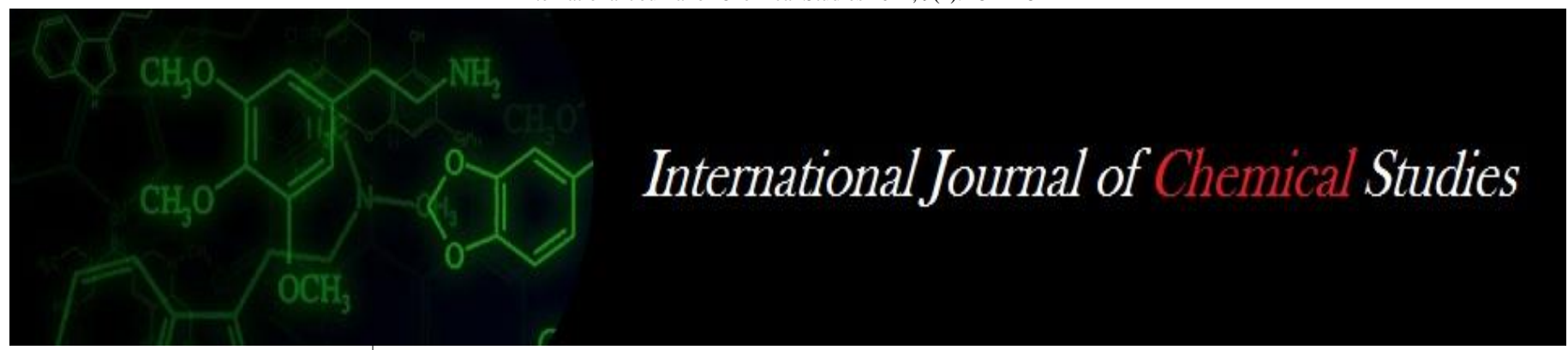

P-ISSN: 2349-8528

E-ISSN: 2321-4902

www.chemijournal.com

IJCS 2021; 9(1): 1541-1544

(C) 2021 IJCS

Received: 07-11-2020

Accepted: 18-12-2020

\section{CH Gunasekhar}

M.Sc. Horticulture, Department of Floriculture and Landscape Architecture, college of horticulture, Mojerla, Sri Konda Laxman Telangana State

Horticultural University, Hyderabad, Telangana, India

Dr. N Seenivasan

Professor and Controller of

Examinations, Sri Konda

Laxman Telangana State

Horticultural University,

Hyderabad, Telangana, India

\section{CNR Santhoshini}

Assistant Professor, Department of Floriculture and Landscape Architecture, college of horticulture, Mojerla, Sri Konda Laxman Telangana State

Horticultural University,

Hyderabad, Telangana, India

\section{Dr. P Gouthami}

Assistant Professor, Department of Plant physiology, college of

horticulture, Mojerla, Sri Konda

Laxman Telangana State

Horticultural University,

Hyderabad, Telangana, India

Dr. G Sathish

Assistant Professor, Department of Agriculture statistics, college of horticulture, Mojerla, Sri

Konda Laxman Telangana State Horticultural University,

Hyderabad, Telangana, India

Corresponding Author: CH Gunasekhar M.Sc. Horticulture, Department of Floriculture and Landscape Architecture, college of horticulture, Mojerla, Sri Konda Laxman Telangana State Horticultural University, Hyderabad, Telangana, India

\section{Influence of bulb treatment with Bio-fertilizers and foliar spray of Bio-stimulants on growth and flowering of tuberose (Polianthes tuberosa L.) cv. Suvasini}

\author{
CH Gunasekhar, Dr. N Seenivasan, CNR Santhoshini, Dr. P Gouthami \\ and Dr. G Sathish
}

DOI: https://doi.org/10.22271/chemi.2021.v9.i1v.11443

\begin{abstract}
This study was conducted to assess the Influence of Bulb treatment with Bio-Fertilizers and foliar spray of Bio-Stimulants on growth and flowering of Tuberose (Polianthes tuberosa L.) cv. Suvasini. The experiment was laid out in Contrast Factorial Randomised Blocked Design consisting of two factors with three and eight levels respectively. Replicated thrice at SKLTSHU, college of horticulture, Mojerla. Tuberose bulbs where treated with three Bio-Fertilizers viz., phosphate solubilizing bacteria (PSB), Azospirillum (AZO) and Potassium solubilizing bacteria (KSB) each $200 \mathrm{~g} / \mathrm{l}$ and they are foliar sprayed by eight Bio-Stimulants gibberellic acid (GA3), salicylic acid (SA), cycocel (CCC) and humic acid (HA) each at $200 \mathrm{ppm}$ and $400 \mathrm{ppm}$. Among bio fertilizers PSB (Phosphate solubilizing bacteria) and among bio stimulants $\mathrm{GA}_{3} 400 \mathrm{ppm}$. while in interaction effect PSB (Phosphate solubilizing bacteria) $200 \mathrm{~g} / \mathrm{l}$ along with $\mathrm{GA}_{3}$ (Gibberellic acid) $400 \mathrm{ppm}$ has resulted significantly best in Plant height $(\mathrm{cm})$, Number of leaves per plant, Length of leaf $(\mathrm{cm})$, Days taken to spike emergence, Spike length $(\mathrm{cm})$, Diameter of spike $(\mathrm{cm})$, Rachis length $(\mathrm{cm})$, Number of florets per spike, Number of spikes per plant, Flower longevity (days), Flower yield or spike yield per hectare (in lakhs), when compared with other treatment combinations and control.
\end{abstract}

Keywords: Tuberose, bulb treatment, PSB, AZO, KSB

\section{Introduction}

Tuberose (Polianthes tuberosa L.) is one of the important bulbous flowering crop. From Mexico, it Spreaded out to the different parts of the world during 16th century (Tiwari and Singh, 2002) [1]. Tuberose is commonly known as 'Rajanigandha' and 'Nelasampengi' in India. Tuberose is commercially grown due to its potential for cut flower, loose flower, long vase life of spikes and pleasant fragrance. (Singh and Kumar, 1999) ${ }^{[2]}$. The flowers remain fresh for quite a long time and withstand distance transportation and occupy a prime place in the flower market (Patel, 2006) ${ }^{[3]}$.

In contrast, to inorganic fertilizer the use of bio-fertilizers and bio-stimulants has been increasing. Bio-fertilizers are biologically active product containing selective strains of microorganism which can contribute nutrients to the plants through microbial activity. These are supplements of chemical fertilizers as they contribute to plant nutrition through biological nitrogen fixation and solubilization of immobile phosphorous (Kumar et al., 2002) ${ }^{[4]}$. Biostimulants are products of natural and organic origin that stimulate plants to achieve their highest growth and yield potential. Bio-stimulants are akin to Bio-fertilizers as they also promote crop growth and yield. The use of Bio-stimulants along with bio-fertilizers could reduce chemical fertilizer use to a large extent and as much as $50 \%$ as they supplement the soil with essential nutrients.

\section{Material and Methods}

The bulbs of tuberose were treated with bio-fertilizers (Phosphate solubilizing bacteria, Azospirillum and Potassium solubilizing bacteria) each $200 \mathrm{~g} / \mathrm{l}$. bulb's were sown on ridge and furrow system with $30 \times 20 \mathrm{~cm}$ spacing between rows and plant respectively. 
After germination they were foliar sprayed by bio-stimulants $\left(\mathrm{GA}_{3}\right.$, Salicylic acid, Cycocel, Humic acid each at $200 \mathrm{ppm}$ and $400 \mathrm{ppm}$ ) There were replicated 3 times for each treatment and experiment was laid out in contrast factorial randomized Block design.

From the treatment plot five plants were selected and observations are recorded on growth and flowering parameters. Plant height $(\mathrm{cm})(30,60$ and 90 days interval), Number of leaves / plants (30,60 and 90 days interval), Length of leaf $(\mathrm{cm})$ (30, 60 and 90 days interval). Flower parameters such as Days taken to spike emergence, Spike length $(\mathrm{cm})$, Diameter of spike $(\mathrm{cm})$, Rachis length $(\mathrm{cm})$, Number of florets per spike, Number of spikes / plants, Flower longevity (days), Flower yield or spike yield / hectare (in lakhs).

\section{Results and Discussion Growth parameters}

(Table-1,2,3) In growth parameters the bio-fertilizer Phosphate solubilizing bacteria (PSB) @ $200 \mathrm{~g} / \mathrm{l}$ was recorded significantly best results in growth parameters where control showing significant difference with the treatments. PSB 200 g/l (Phosphate solubilizing bacteria) recorded highest Plant height at 30, 60 and 90 days with $11.84 \mathrm{~cm}, 32.15 \mathrm{~cm}$ and $44.81 \mathrm{~cm}$ respectively. Also recorded highest number of leaves / plants recorded at 30, 60 and 90 days with 5.56, 10.73 and 18.58 respectively. PSB recorded highest length of leaf at 30,60 and 90 days with $9.62 \mathrm{~cm}, 18.84 \mathrm{~cm}$ and $28.10 \mathrm{~cm}$ respectively. When compared with other bio-fertilizers and control treatment. (Table-1,2,3) The bio-stimulants $\mathrm{GA}_{3} 400$ ppm was recorded significantly best results in growth parameters. where control showing significant difference with the treatments. $\mathrm{GA}_{3} 400 \mathrm{ppm}$ recorded highest Plant height at 30,60 and 90 days with $13.23 \mathrm{~cm}, 33.71 \mathrm{~cm}$ and $46.52 \mathrm{~cm}$ respectively. Also recorded highest number of leaves / plants recorded at 30, 60 and 90 days with 5.92, 11.95 and 20.28 respectively. $\mathrm{GA}_{3} 400 \mathrm{ppm}$ recorded highest length of leaf at 30,60 and 90 days with $10.43 \mathrm{~cm}, 19.69 \mathrm{~cm}$ and $28.30 \mathrm{~cm}$ respectively. When compared with other bio-stimulants and control treatment. (Table-1,2,3) Coming to the interaction effect of bio-fertilizers and bio-stimulants where the combination of PSB $200 \mathrm{~g} / \mathrm{l}$ (Phosphate solubilizing bacteria) $+\mathrm{GA}_{3} 400$ ppm was recorded significantly best results in growth parameters. where control showing significant difference with the treatments. PSB $200 \mathrm{~g} / \mathrm{l}$ (Phosphate solubilizing bacteria) $+\mathrm{GA}_{3} 400 \mathrm{ppm}$ recorded highest Plant height at 30, 60 and 90 days with $16.08 \mathrm{~cm}, 38.00 \mathrm{~cm}$ and $50.13 \mathrm{~cm}$ respectively. Also recorded highest number of leaves / plants recorded at 30,60 and 90 days with 7.08, 13.50 and 22.99 respectively. PSB $200 \mathrm{~g} / \mathrm{l}$ (Phosphate solubilizing bacteria) $+\mathrm{GA}_{3} 400 \mathrm{ppm}$ recorded highest length of leaf at 30,60 and 90 days with $11.95 \mathrm{~cm}, 21.68 \mathrm{~cm}$ and $30.95 \mathrm{~cm}$ respectively. When compared with other treatment combinations and control treatment.

\section{Floral parameters}

(Table-4,5,6) In floral parameters the bio-fertilizer Phosphate solubilizing bacteria (PSB) @ $200 \mathrm{~g} / \mathrm{l}$ was recorded significantly best results in floral parameters. Where control showing significant difference with the treatment. where it has taken lowest number of days for first spike emergence (153.16 days), highest Spike length $(65.39 \mathrm{~cm})$, maximum diameter of spike $(0.81 \mathrm{~cm})$, maximum Rachis length $(19.46$ $\mathrm{cm}$ ), highest number of florets per spike (29.08), highest number of spikes / plants (1.93), maximum flower longevity (18.88 days), highest flower yield or spike yield / hectare (0.15 in lakhs). When compared with other bio-fertilizers and control treatment.

(Table-4,5,6) The bio-stimulant $\mathrm{GA}_{3} 400$ ppm was recorded significantly best results in floral parameters. Where control showing significant difference with the treatment. where it has taken lowest number of days for first spike emergence (149.72 days), highest Spike length $(66.04 \mathrm{~cm})$, maximum diameter of spike $(0.85 \mathrm{~cm})$, maximum Rachis length $(20.89$ $\mathrm{cm}$ ), highest number of florets per spike (31.08), highest number of spikes / plants (2.23), maximum flower longevity (20.24 days), highest flower yield or spike yield / hectare (0.17 in lakhs). When compared with other bio-stimulants and control treatment.

(Table-4,5,6) Coming to the interaction effect of biofertilizers and bio-stimulants where the combination of PSB $200 \mathrm{~g} / \mathrm{l}$ (Phosphate solubilizing bacteria) $+\mathrm{GA}_{3} 400 \mathrm{ppm}$ was recorded significantly best results in floral parameters. where control showing significant difference with the treatments. where it has taken lowest number of days for first spike emergence (136.24 days), highest Spike length $(70.53 \mathrm{~cm})$, maximum diameter of spike $(0.94 \mathrm{~cm})$, maximum Rachis length $(24.28 \mathrm{~cm})$, highest number of florets per spike (35.62), highest number of spikes / plants (2.59), maximum flower longevity (21.91 days), highest flower yield or spike yield / hectare (0.24 in lakhs). When compared with other treatment combinations and control treatment.

Table 1: Influence of Bulb treatment with Bio-fertilizers and foliar spray of Bio-stimulants on plant height at 30, 60 and 90 days in tuberose cv. Suvasini

\begin{tabular}{|c|c|c|c|c|c|c|c|c|c|c|c|c|}
\hline \multirow{3}{*}{$\begin{array}{l}\text { Bio stimulants } \\
\text { (Factor - 2) }\end{array}$} & \multicolumn{12}{|c|}{ Bio fertilizers (Factor - 1) } \\
\hline & \multicolumn{4}{|c|}{30 days } & \multicolumn{4}{|c|}{60 days } & \multicolumn{4}{|c|}{90 days } \\
\hline & $\mathbf{P}_{1}$ & $\mathbf{P}_{2}$ & $\mathbf{P}_{3}$ & mean & $\mathbf{P}_{1}$ & $\mathbf{P}_{2}$ & $\mathbf{P}_{3}$ & mean & $\mathbf{P}_{1}$ & $\mathbf{P}_{2}$ & $\mathbf{P}_{3}$ & mean \\
\hline $\mathrm{S}_{1}-\mathrm{GA}_{3} 200 \mathrm{ppm}$ & 15.73 & 10.68 & 11.46 & 12.62 & 35.28 & 30.28 & 34.72 & 33.43 & 48.80 & 42.13 & 48.60 & 46.51 \\
\hline $\mathrm{S}_{2}-\mathrm{GA}_{3} 400 \mathrm{ppm}$ & 16.08 & 9.41 & 14.20 & 13.23 & 38.00 & 27.87 & 35.27 & 33.71 & 50.13 & 44.97 & 44.47 & 46.52 \\
\hline $\mathrm{S}_{3}-\mathrm{SA} 200 \mathrm{ppm}$ & 9.81 & 9.63 & 10.46 & 9.97 & 29.52 & 33.67 & 27.56 & 30.25 & 41.87 & 42.23 & 41.50 & 41.87 \\
\hline $\mathrm{S}_{4}-\mathrm{SA} 400 \mathrm{ppm}$ & 10.48 & 10.44 & 11.26 & 10.73 & 34.34 & 27.33 & 30.23 & 30.63 & 42.90 & 41.87 & 42.67 & 42.48 \\
\hline $\mathrm{S}_{5}-\mathrm{CCC} 200 \mathrm{ppm}$ & 12.78 & 9.57 & 9.92 & 10.76 & 30.74 & 28.02 & 28.08 & 28.95 & 47.07 & 41.80 & 42.70 & 43.86 \\
\hline $\mathrm{S}_{6}-\mathrm{CCC} 400 \mathrm{ppm}$ & 9.54 & 11.32 & 9.39 & 10.08 & 29.14 & 30.22 & 25.61 & 28.32 & 42.30 & 44.63 & 40.13 & 42.35 \\
\hline $\mathrm{S}_{7}-\mathrm{HA} 200 \mathrm{ppm}$ & 10.06 & 10.19 & 10.4 & 10.22 & 32.06 & 29.3 & 28.3 & 29.89 & 43.20 & 42.33 & 41.97 & 42.50 \\
\hline $\mathrm{S}_{8}-\mathrm{HA} 400 \mathrm{ppm}$ & 10.27 & 9.81 & 11.00 & 10.36 & 28.09 & 33.16 & 26.69 & 29.31 & 42.20 & 43.40 & 42.53 & 42.71 \\
\hline mean & 11.84 & 10.13 & 11.01 & & 32.15 & 29.98 & 29.56 & & 44.81 & 42.92 & 43.07 & \\
\hline control & \multicolumn{4}{|c|}{8.11} & \multicolumn{4}{|c|}{25.40} & \multicolumn{4}{|c|}{40.57} \\
\hline & $\mathrm{P}$ & $\mathrm{S}$ & $\mathrm{P} \times \mathrm{S}$ & control & $\mathrm{P}$ & $\mathrm{S}$ & $\mathrm{P} \times \mathrm{S}$ & control & $\mathrm{P}$ & $\mathrm{S}$ & $\mathrm{P} \times \mathrm{S}$ & control \\
\hline S.Em \pm & 0.03 & 0.09 & 0.26 & 0.46 & 0.07 & 0.18 & 0.53 & 1.94 & 0.09 & 0.24 & 0.73 & 1.29 \\
\hline LSD@5\% & $0.09^{*}$ & $0.25^{*}$ & $0.75^{*}$ & $0.93^{*}$ & $0.19^{*}$ & $0.50^{*}$ & $1.51^{*}$ & $1.89^{*}$ & $0.26^{*}$ & $0.69^{*}$ & $2.07^{*}$ & $2.58^{*}$ \\
\hline
\end{tabular}

$\mathrm{GA}_{3}=$ Gibberellic acid, $\mathrm{SA}=$ Salicylic acid, $\mathrm{CCC}=$ Cycocel, $\mathrm{HA}=$ Humic acid, $\left(\mathrm{P}_{1}\right) \mathrm{PSB}=$ Phosphate solubilizing bacteria, $\left(\mathrm{P}_{2}\right)$ AZO $=$ Azospirillum, $\left(\mathrm{P}_{3}\right) \mathrm{KSB}=$ Potassium solubilizing Bacteria 
Table 2: Influence of Bulb treatment with Bio-fertilizers and foliar spray of Bio-stimulants on number of leaves per plant at 30, 60 and 90 days in tuberose cv. Suvasini

\begin{tabular}{|c|c|c|c|c|c|c|c|c|c|c|c|c|}
\hline \multirow{3}{*}{$\begin{array}{l}\text { Bio stimulants } \\
\text { (Factor - 2) }\end{array}$} & \multicolumn{12}{|c|}{ Bio fertilizers (Factor - 1) } \\
\hline & \multicolumn{4}{|c|}{30 days } & \multicolumn{4}{|c|}{60 days } & \multicolumn{4}{|c|}{90 days } \\
\hline & $\mathbf{P}_{1}$ & $\mathbf{P}_{2}$ & $\mathbf{P}_{3}$ & mean & $\mathbf{P}_{1}$ & $\mathbf{P}_{2}$ & $\mathbf{P}_{3}$ & mean & $\mathbf{P}_{1}$ & $\mathbf{P}_{2}$ & $\mathbf{P}_{3}$ & mean \\
\hline$S_{1}-G_{3} 200 \mathrm{ppm}$ & 6.90 & 4.14 & 6.50 & 5.85 & 12.90 & 8.68 & 12.34 & 11.31 & 21.11 & 18.99 & 15.88 & 18.66 \\
\hline $\mathrm{S}_{2}-\mathrm{GA}_{3} 400 \mathrm{ppm}$ & 7.08 & 3.95 & 6.73 & 5.92 & 13.50 & 10.15 & 12.20 & 11.95 & 22.99 & 20.94 & 16.90 & 20.28 \\
\hline $\mathrm{S}_{3}-\mathrm{SA} 200 \mathrm{ppm}$ & 5.86 & 4.12 & 5.53 & 5.17 & 10.74 & 8.71 & 11.40 & 10.28 & 19.01 & 20.17 & 16.36 & 18.51 \\
\hline $\mathrm{S}_{4}-\mathrm{SA} 400 \mathrm{ppm}$ & 4.12 & 5.79 & 4.35 & 4.75 & 9.19 & 11.39 & 8.38 & 9.65 & 16.38 & 16.76 & 18.87 & 17.34 \\
\hline $\mathrm{S}_{5}-\mathrm{CCC} 200 \mathrm{ppm}$ & 6.08 & 3.84 & 6.83 & 5.58 & 10.19 & 8.68 & 9.87 & 9.58 & 19.25 & 19.99 & 16.18 & 18.47 \\
\hline $\mathrm{S}_{6}-\mathrm{CCC} 400 \mathrm{ppm}$ & 4.87 & 4.05 & 4.11 & 4.34 & 9.14 & 8.94 & 8.36 & 8.81 & 16.81 & 15.11 & 15.98 & 15.97 \\
\hline $\mathrm{S}_{7}-\mathrm{HA} 200 \mathrm{ppm}$ & 4.94 & 4.71 & 4.79 & 4.81 & 10.07 & 10.54 & 8.56 & 9.72 & 16.33 & 16.36 & 16.90 & 16.53 \\
\hline $\mathrm{S}_{8}-\mathrm{HA} 400 \mathrm{ppm}$ & 4.62 & 5.75 & 3.98 & 4.78 & 10.07 & 10.47 & 8.75 & 9.76 & 16.78 & 15.96 & 18.31 & 17.02 \\
\hline mean & 5.56 & 4.54 & 5.35 & & 10.73 & 9.70 & 9.98 & & 18.58 & 18.04 & 16.92 & \\
\hline control & \multicolumn{4}{|c|}{4.13} & \multicolumn{4}{|c|}{8.06} & \multicolumn{4}{|c|}{15.04} \\
\hline & $\mathrm{P}$ & $\mathrm{S}$ & $\mathrm{P} \times \mathrm{S}$ & control & $\mathrm{P}$ & $\mathrm{S}$ & $\mathrm{P} \times \mathrm{S}$ & control & $\mathrm{P}$ & $\mathrm{S}$ & $\mathrm{P} \times \mathrm{S}$ & control \\
\hline S.Em \pm & 0.02 & 0.06 & 0.17 & 0.30 & 0.02 & 0.06 & 0.18 & 0.31 & 0.04 & 0.10 & 0.30 & 0.53 \\
\hline LSD@5\% & $0.06^{*}$ & $0.16^{*}$ & $0.49^{*}$ & $0.61^{*}$ & $0.06^{*}$ & $0.17^{*}$ & $0.50^{*}$ & $0.62^{*}$ & $0.11^{*}$ & $0.28 *$ & $0.85^{*}$ & $1.06^{*}$ \\
\hline
\end{tabular}

Table 3: Influence of Bulb treatment with Bio-fertilizers and foliar spray of Bio-stimulants on length of leaves (cm) at 30, 60 and 90 days in tuberose cv. Suvasini

\begin{tabular}{|c|c|c|c|c|c|c|c|c|c|c|c|c|}
\hline \multirow{3}{*}{$\begin{array}{l}\text { Bio stimulants } \\
\text { (Factor - 2) }\end{array}$} & \multicolumn{12}{|c|}{ Bio fertilizers (Factor - 1) } \\
\hline & \multicolumn{4}{|c|}{ 30 days } & \multicolumn{4}{|c|}{60 days } & \multicolumn{4}{|c|}{90 days } \\
\hline & $\mathbf{P}_{1}$ & $\mathbf{P}_{2}$ & $\mathbf{P}_{3}$ & mean & $\mathbf{P}_{1}$ & $\mathbf{P}_{2}$ & $\mathbf{P}_{3}$ & mean & $\mathbf{P}_{1}$ & $\mathbf{P}_{2}$ & $\mathbf{P}_{3}$ & mean \\
\hline $\mathrm{S}_{1}-\mathrm{GA}_{3} 200 \mathrm{ppm}$ & 11.46 & 7.78 & 9.32 & 9.52 & 20.45 & 16.04 & 18.80 & 18.43 & 29.90 & 25.33 & 29.15 & 28.13 \\
\hline $\mathrm{S}_{2}-\mathrm{GA}_{3} 400 \mathrm{ppm}$ & 11.95 & 8.56 & 10.77 & 10.43 & 21.68 & 17.40 & 19.99 & 19.69 & 30.95 & 26.35 & 27.59 & 28.30 \\
\hline $\mathrm{S}_{3}-\mathrm{SA} 200 \mathrm{ppm}$ & 9.85 & 8.15 & 9.81 & 9.27 & 18.74 & 16.30 & 19.67 & 18.24 & 27.46 & 25.29 & 27.30 & 26.68 \\
\hline $\mathrm{S}_{4}-\mathrm{SA} 400 \mathrm{ppm}$ & 8.29 & 9.80 & 8.26 & 8.78 & 17.73 & 19.43 & 16.00 & 17.72 & 29.17 & 24.91 & 25.50 & 26.53 \\
\hline $\mathrm{S}_{5}-\mathrm{CCC} 200 \mathrm{ppm}$ & 10.48 & 7.91 & 9.13 & 9.17 & 16.05 & 20.28 & 16.60 & 17.64 & 26.48 & 27.17 & 23.92 & 25.86 \\
\hline $\mathrm{S}_{6}-\mathrm{CCC} 400 \mathrm{ppm}$ & 8.27 & 9.33 & 7.71 & 8.44 & 17.64 & 19.15 & 15.30 & 17.36 & 25.74 & 27.72 & 26.03 & 26.50 \\
\hline $\mathrm{S}_{7}-\mathrm{HA} 200 \mathrm{ppm}$ & 8.06 & 9.54 & 8.14 & 8.58 & 20.43 & 15.44 & 19.03 & 18.30 & 27.17 & 28.80 & 25.79 & 27.25 \\
\hline $\mathrm{S}_{8}-\mathrm{HA} 400 \mathrm{ppm}$ & 8.6 & 10.06 & 8.18 & 8.95 & 17.99 & 19.38 & 16.18 & 17.85 & 27.96 & 29.49 & 25.73 & 27.73 \\
\hline mean & 9.62 & 8.89 & 8.92 & & 18.84 & 17.93 & 17.70 & & 28.10 & 26.88 & 26.38 & \\
\hline \multirow[t]{2}{*}{ control } & \multicolumn{4}{|c|}{7.93} & \multicolumn{4}{|c|}{14.82} & \multicolumn{4}{|c|}{24.51} \\
\hline & $\mathrm{P}$ & $\mathrm{S}$ & $\mathrm{P} \times \mathrm{S}$ & control & $\mathrm{P}$ & $\mathrm{S}$ & $\mathrm{P} \times \mathrm{S}$ & control & $\mathrm{P}$ & $\mathrm{S}$ & $\mathrm{P} \times \mathrm{S}$ & control \\
\hline S.Em \pm & 0.02 & 0.06 & 0.17 & 0.29 & 0.04 & 0.10 & 0.30 & 0.53 & 0.06 & 0.15 & 0.45 & 0.80 \\
\hline LSD@5\% & $0.06^{*}$ & $0.16^{*}$ & $0.47 *$ & $0.59 *$ & $0.11^{*}$ & $0.29 *$ & $0.86^{*}$ & $1.07 *$ & $0.16^{*}$ & $0.43 *$ & $1.28 *$ & $1.60 *$ \\
\hline
\end{tabular}

$\mathrm{GA}_{3}=$ Gibberellic acid, SA = Salicylic acid, CCC $=$ Cycocel, $\mathrm{HA}=$ Humic acid, $\left(\mathrm{P}_{1}\right) \mathrm{PSB}=$ Phosphate solubilizing bacteria, $\left(\mathrm{P}_{2}\right) \mathrm{AZO}=$ Azospirillum, $\left(\mathrm{P}_{3}\right) \mathrm{KSB}=$ Potassium solubilizing Bacteria

Table 4: Influence of Bulb treatment with Bio-fertilizers and Foliar spray of Bio-stimulants on spike emergence (days), spike length (cm), diameter of spike $(\mathrm{cm})$ in tuberose cv. Suvasini

\begin{tabular}{|c|c|c|c|c|c|c|c|c|c|c|c|c|}
\hline \multirow{3}{*}{$\begin{array}{c}\text { Bio stimulants } \\
\text { (Factor - 2) }\end{array}$} & \multicolumn{12}{|c|}{ Bio fertilizers (Factor - 1) } \\
\hline & \multicolumn{4}{|c|}{ spike emergence (days) } & \multicolumn{4}{|c|}{ spike length $(\mathrm{cm})$} & \multicolumn{4}{|c|}{ diameter of spike $(\mathrm{cm})$} \\
\hline & $\mathbf{P}_{1}$ & $\mathbf{P}_{2}$ & $\mathbf{P}_{3}$ & mean & $\mathbf{P}_{1}$ & $\mathbf{P}_{2}$ & $\mathbf{P}_{3}$ & mean & $\mathbf{P}_{1}$ & $\mathbf{P}_{2}$ & $\mathbf{P}_{3}$ & mean \\
\hline $\mathrm{S}_{1}-\mathrm{GA}_{3} 200 \mathrm{ppm}$ & 143.37 & 161.64 & 145.28 & 150.10 & 69.10 & 59 & 66.73 & 64.94 & 0.90 & 0.72 & 0.89 & 0.84 \\
\hline $\mathrm{S}_{2}-\mathrm{GA}_{3} 400 \mathrm{ppm}$ & 136.24 & 163.33 & 149.58 & 149.72 & 70.53 & 59 & 68.60 & 66.04 & 0.94 & 0.86 & 0.74 & 0.85 \\
\hline $\mathrm{S}_{3}-\mathrm{SA} 200 \mathrm{ppm}$ & 156.00 & 161.55 & 163.90 & 160.48 & 65.23 & 58.4 & 68.17 & 63.93 & 0.74 & 0.71 & 0.72 & 0.72 \\
\hline $\mathrm{S}_{4}-\mathrm{SA} 400 \mathrm{ppm}$ & 161.24 & 151.61 & 159.39 & 157.41 & 64.90 & 59.8 & 58.37 & 61.02 & 0.81 & 0.74 & 0.88 & 0.81 \\
\hline $\mathrm{S}_{5}-\mathrm{CCC} 200 \mathrm{ppm}$ & 157.68 & 158.58 & 165.23 & 160.50 & 62.63 & 58.47 & 58.63 & 59.91 & 0.76 & 0.75 & 0.71 & 0.74 \\
\hline $\mathrm{S}_{6}-\mathrm{CCC} 400 \mathrm{ppm}$ & 157.39 & 164.45 & 161.70 & 161.18 & 63.5 & 60.13 & 57.37 & 60.33 & 0.87 & 0.71 & 0.79 & 0.79 \\
\hline $\mathrm{S}_{7}-\mathrm{HA} 200 \mathrm{ppm}$ & 158.30 & 164.41 & 146.68 & 156.46 & 65.53 & 68.83 & 58.37 & 64.24 & 0.70 & 0.71 & 0.69 & 0.70 \\
\hline $\mathrm{S}_{8}-\mathrm{HA} 400 \mathrm{ppm}$ & 155.05 & 154.43 & 163.67 & 157.72 & 61.7 & 68.60 & 58.03 & 62.78 & 0.74 & 0.84 & 0.74 & 0.77 \\
\hline mean & 153.16 & 160.00 & 156.93 & & 65.39 & 61.53 & 61.78 & & 0.81 & 0.76 & 0.77 & \\
\hline control & \multicolumn{4}{|c|}{163.97} & \multicolumn{4}{|c|}{59.23} & \multicolumn{4}{|c|}{0.65} \\
\hline & $\mathrm{P}$ & $\mathrm{S}$ & $\mathrm{P} \times \mathrm{S}$ & control & $\mathrm{P}$ & $\mathrm{S}$ & $\mathrm{P} \times \mathrm{S}$ & control & $\mathrm{P}$ & $\mathrm{S}$ & $\mathrm{P} \times \mathrm{S}$ & control \\
\hline S.Em \pm & 0.32 & 0.87 & 2.60 & NS & 0.11 & 0.28 & 0.84 & 1.49 & 0.002 & 0.004 & 0.013 & 0.02 \\
\hline LSD@5\% & $0.92 *$ & $2.46^{*}$ & $7.38 *$ & NS & $0.30 *$ & $0.80 *$ & $2.40^{*}$ & $3.00 *$ & $0.005^{*}$ & $0.012 *$ & $0.037 *$ & $0.05 *$ \\
\hline
\end{tabular}

$\mathrm{GA}_{3}=$ Gibberellic acid, SA = Salicylic acid, CCC = Cycocel, HA = Humic acid, $\left(\mathrm{P}_{1}\right)$ PSB = Phosphate solubilizing bacteria, $\left(\mathrm{P}_{2}\right)$ AZO = Azospirillum, $\left(\mathrm{P}_{3}\right) \mathrm{KSB}=$ Potassium solubilizing Bacteria 
Table 5: Influence of Bulb treatment with Bio-fertilizers and foliar spray of Bio-stimulants on rachis length (cm), number of florets per spike, number of spikes per plant in tuberose cv. Suvasini

\begin{tabular}{|c|c|c|c|c|c|c|c|c|c|c|c|c|}
\hline \multirow{3}{*}{$\begin{array}{l}\text { Bio stimulants } \\
(\text { Factor - 2) }\end{array}$} & \multicolumn{12}{|c|}{ Bio fertilizers (Factor - 1) } \\
\hline & \multicolumn{4}{|c|}{ rachis length $(\mathrm{cm})$} & \multicolumn{4}{|c|}{ number of florets per spike } & \multicolumn{4}{|c|}{ number of spikes per plant } \\
\hline & $\mathbf{P}_{1}$ & $\mathbf{P}_{2}$ & $\mathbf{P}_{3}$ & mean & $\mathbf{P}_{1}$ & $\mathbf{P}_{2}$ & $\mathbf{P}_{3}$ & mean & $\mathbf{P}_{1}$ & $\mathbf{P}_{2}$ & $\mathbf{P}_{3}$ & mean \\
\hline $\mathrm{S}_{1}-\mathrm{GA}_{3} 200 \mathrm{ppm}$ & 23.55 & 16.11 & 21.09 & 20.25 & 34.32 & 24.45 & 30.09 & 29.62 & 2.14 & 2.09 & 1.95 & 2.06 \\
\hline $\mathrm{S}_{2}-\mathrm{GA}_{3} 400 \mathrm{ppm}$ & 24.28 & 16.24 & 22.15 & 20.89 & 35.62 & 24.79 & 32.83 & 31.08 & 2.59 & 2.02 & 2.07 & 2.23 \\
\hline $\mathrm{S}_{3}-\mathrm{SA} 200 \mathrm{ppm}$ & 17.58 & 19.41 & 14.8 & 17.26 & 23.11 & 33.74 & 20.16 & 25.67 & 1.34 & 1.76 & 2.04 & 1.71 \\
\hline $\mathrm{S}_{4}-\mathrm{SA} 400 \mathrm{ppm}$ & 16.49 & 20.69 & 15.01 & 17.40 & 34.29 & 32.14 & 20.79 & 29.07 & 1.92 & 1.94 & 1.87 & 1.91 \\
\hline $\mathrm{S}_{5}-\mathrm{CCC} 200 \mathrm{ppm}$ & 19.66 & 14.89 & 22.70 & 19.08 & 26.94 & 23.42 & 22.93 & 24.43 & 1.74 & 1.24 & 1.61 & 1.53 \\
\hline $\mathrm{S}_{6}-\mathrm{CCC} 400 \mathrm{ppm}$ & 21.94 & 14.56 & 23.14 & 19.88 & 22.99 & 29.67 & 20.39 & 24.35 & 1.92 & 1.74 & 1.34 & 1.67 \\
\hline $\mathrm{S}_{7}-\mathrm{HA} 200 \mathrm{ppm}$ & 16.16 & 19.65 & 14.28 & 16.70 & 29.64 & 25.38 & 31.64 & 28.89 & 1.78 & 1.42 & 1.93 & 1.71 \\
\hline $\mathrm{S}_{8}-\mathrm{HA} 400 \mathrm{ppm}$ & 15.98 & 22.55 & 14.43 & 17.65 & 25.73 & 32.28 & 23.16 & 27.06 & 1.98 & 1.76 & 1.86 & 1.87 \\
\hline mean & 19.46 & 18.01 & 18.45 & & 29.08 & 28.23 & 25.25 & & 1.93 & 1.75 & 1.83 & \\
\hline control & \multicolumn{4}{|c|}{12.58} & \multicolumn{4}{|c|}{21.52} & \multicolumn{4}{|c|}{1.97} \\
\hline & $\mathrm{P}$ & $\mathrm{S}$ & $\mathrm{P} \times \mathrm{S}$ & control & $\mathrm{P}$ & $\mathrm{S}$ & $\mathrm{P} \times \mathrm{S}$ & control & $\mathrm{P}$ & $\mathrm{S}$ & $\mathrm{P} \times \mathrm{S}$ & control \\
\hline S.Em \pm & 0.04 & 0.10 & 0.31 & 0.55 & 0.10 & 0.27 & 0.81 & 1.43 & 0.01 & 0.03 & 0.10 & NS \\
\hline LSD@5\% & $0.11 *$ & $0.29 *$ & $0.88^{*}$ & $1.11^{*}$ & $0.29 *$ & $0.77 *$ & $2.30 *$ & $2.88^{*}$ & $0.03 *$ & $0.09 *$ & $0.27 *$ & NS* \\
\hline
\end{tabular}

$\mathrm{GA}_{3}=$ Gibberellic acid, SA $=$ Salicylic acid, CCC $=$ Cycocel, $\mathrm{HA}=$ Humic acid, $\left(\mathrm{P}_{1}\right) \mathrm{PSB}=$ Phosphate solubilizing bacteria, $\left(\mathrm{P}_{2}\right) \mathrm{AZO}=$ Azospirillum, $\left(\mathrm{P}_{3}\right) \mathrm{KSB}=$ Potassium solubilizing Bacteria

Table 6: Influence of Bulb treatment with Bio-fertilizers and Foliar spray of Bio-stimulants on number of spikes per plant, flower longevity (days), Flower yield per hectare (in lakhs) in tuberose cv. Suvasini

\begin{tabular}{|c|c|c|c|c|c|c|c|c|c|c|c|c|}
\hline \multirow{3}{*}{$\begin{array}{l}\text { Bio stimulants } \\
(\text { Factor - 2) }\end{array}$} & \multicolumn{12}{|c|}{ Bio fertilizers (Factor - 1) } \\
\hline & \multicolumn{4}{|c|}{ number of spikes per plant } & \multicolumn{4}{|c|}{ flower longevity (days) } & \multicolumn{4}{|c|}{ Flower yield per hectare (in lakhs) } \\
\hline & $\mathbf{P}_{1}$ & $\mathbf{P}_{2}$ & $\mathbf{P}_{3}$ & mean & $\mathbf{P}_{1}$ & $\mathbf{P}_{2}$ & $\mathbf{P}_{3}$ & mean & $\mathbf{P}_{1}$ & $\mathbf{P}_{2}$ & $\mathbf{P}_{3}$ & mean \\
\hline $\mathrm{S}_{1}-\mathrm{GA}_{3} 200 \mathrm{ppm}$ & 2.14 & 2.09 & 1.95 & 2.06 & 21.19 & 15.47 & 19.36 & 18.67 & 0.21 & 0.11 & 0.11 & 0.14 \\
\hline $\mathrm{S}_{2}-\mathrm{GA}_{3} 400 \mathrm{ppm}$ & 2.59 & 2.02 & 2.07 & 2.23 & 21.91 & 19.82 & 18.99 & 20.24 & 0.24 & 0.16 & 0.1 & 0.17 \\
\hline $\mathrm{S}_{3}-\mathrm{SA} 200 \mathrm{ppm}$ & 1.34 & 1.76 & 2.04 & 1.71 & 20.00 & 15.23 & 13.83 & 16.35 & 0.09 & 0.11 & 0.15 & 0.12 \\
\hline $\mathrm{S}_{4}-\mathrm{SA} 400 \mathrm{ppm}$ & 1.92 & 1.94 & 1.87 & 1.91 & 17.18 & 13.85 & 20.64 & 17.22 & 0.12 & 0.08 & 0.17 & 0.12 \\
\hline $\mathrm{S}_{5}-\mathrm{CCC} 200 \mathrm{ppm}$ & 1.74 & 1.24 & 1.61 & 1.53 & 19.13 & 14.75 & 21.10 & 18.33 & 0.11 & 0.06 & 0.08 & 0.08 \\
\hline $\mathrm{S}_{6}-\mathrm{CCC} 400 \mathrm{ppm}$ & 1.92 & 1.74 & 1.34 & 1.67 & 20.11 & 15.34 & 14.83 & 16.76 & 0.11 & 0.08 & 0.08 & 0.09 \\
\hline $\mathrm{S}_{7}-\mathrm{HA} 200 \mathrm{ppm}$ & 1.78 & 1.42 & 1.93 & 1.71 & 14.44 & 19.06 & 13.53 & 15.68 & 0.13 & 0.09 & 0.18 & 0.13 \\
\hline $\mathrm{S}_{8}-\mathrm{HA} 400 \mathrm{ppm}$ & 1.98 & 1.76 & 1.86 & 1.87 & 17.08 & 19.73 & 12.81 & 16.54 & 0.16 & 0.11 & 0.13 & 0.13 \\
\hline mean & 1.93 & 1.75 & 1.83 & & 18.88 & 16.66 & 16.89 & & 0.15 & 0.10 & 0.13 & \\
\hline control & \multicolumn{4}{|c|}{1.97} & \multicolumn{4}{|c|}{12.22} & \multicolumn{4}{|c|}{0.08} \\
\hline & $\mathrm{P}$ & $\mathrm{S}$ & $\mathrm{P} \times \mathrm{S}$ & control & $\mathrm{P}$ & $\mathrm{S}$ & $\mathrm{P} \times \mathrm{S}$ & control & $\mathrm{P}$ & $\mathrm{S}$ & $\mathrm{P} \times \mathrm{S}$ & control \\
\hline S.Em \pm & 0.01 & 0.03 & 0.10 & NS & 0.04 & 0.10 & 0.30 & 0.53 & 0.001 & 0.003 & 0.009 & 0.02 \\
\hline LSD@5\% & $0.03 *$ & $0.09 *$ & $0.27 *$ & NS* & $0.11 *$ & $0.29 *$ & $0.86^{*}$ & $1.07 *$ & $0.003 *$ & $0.009^{*}$ & $0.026^{*}$ & $0.03 *$ \\
\hline
\end{tabular}

$\mathrm{GA}_{3}=$ Gibberellic acid, SA = Salicylic acid, CCC = Cycocel, HA = Humic acid, $\left(\mathrm{P}_{1}\right)$ PSB = Phosphate solubilizing bacteria, $\left(\mathrm{P}_{2}\right)$ AZO $=$ Azospirillum, $\left(\mathrm{P}_{3}\right) \mathrm{KSB}=$ Potassium solubilizing Bacteria

\section{Conclusion}

From the results, it can be concluded that among the biofertilizers Phosphate solubilizing bacteria (PSB) 200g/l, in bio-stimulants $\mathrm{GA}_{3} 400 \mathrm{ppm}$ while in interaction effect of bio-fertilizers and bio-stimulants where the combination of PSB $200 \mathrm{~g} / \mathrm{l}$ (Phosphate solubilizing bacteria) $+\mathrm{GA}_{3} 400 \mathrm{ppm}$ has significantly resulted best in Growth parameters such as, Plant height $(\mathrm{cm})(30,60$ and 90 days interval), Number of leaves / plants (30,60 and 90 days interval), Length of leaf (cm) (30, 60 and 90 days interval) and Floral parameters such as Days taken to spike emergence, Spike length $(\mathrm{cm})$, Diameter of spike $(\mathrm{cm})$, Rachis length $(\mathrm{cm})$, Number of florets per spike, Number of spikes / plants, Flower longevity (days), Flower yield or spike yield / hectare (in lakhs).

\section{References}

1. Tiwari JK, Singh RP. Effect of pre-planting GA3 treatment on tuberose. Journal of Ornamental Horticulture 2002;5(2):44-45.

2. Singh PV, Kumar M. Effect of spacing, depth and time of planting on growth, flowering and bulb production of tuberose Cv. Single. Horticultural Science 1999;2(2):127130.

3. Patel MM, Parmer PB, Parmer BR. Effect of nitrogen, phosphorus and spacing on growth and flowering in tuberose Cv. Single. Journal of Ornamental Horticulture 2006;9(4):286-289.

4. Kumar P. Response of bio-fertilizer and $\mathrm{GA}_{3}$ on growth and yield of China aster (Callistephus chinensis L.). $\mathrm{Ph} . \mathrm{D}$. thesis, submitted in division of floriculture and landscaping, IARI, New Delhi. Narayan 2002. 